\title{
Intramedullary nailing for fibrous dysplasia of lower limbs
}

\author{
XIAOQI ZHANG，XIFU SHANG，YAOFEI WANG，RUI HE and GUOGUANG SHI \\ Department of Orthopedic Surgery, Anhui Provincial Hospital Affiliated to Anhui Medical University, \\ Hefei, Anhui 230001, P.R. China
}

Received February 8, 2012; Accepted May 29, 2012

DOI: $10.3892 / \mathrm{ol} .2012 .752$

\begin{abstract}
Fibrous dysplasia (FD) of the bone is rare and self-limiting. However, lesion expansion may occur, causing pain, deformity and pathological fracture, in which case surgery is occasionally required. Indicators of FD have not been previously described, although there are several surgical procedures reported (curettage, curettage and graft, and internal fixation). In this study we discuss whether intramedullary nailing of these lesions could result in more favorable outcomes in correcting deformities, including the prevention of secondary fractures and maintenance of the bone, compared to other internal fixation methods. A total of 39 patients with FD of the bone treated with intramedullary nailing were retrospectively analyzed. The surgical procedures involved curettage, grafting and intramedullary nailing. No infection, thromboembolism or other notable complications occurred. The patients resumed full activities of daily living. At the last follow-up, 33 patients presented no pain and seven patients had occasional mild pain. The clinical score according to the modified criteria of Guille improved from an average of 4.4 points prior to surgery to an average of 8 points following surgery. The neck shaft angle of the femur improved from an average of $90^{\circ}$ prior to surgery to $125^{\circ}$ following surgery. Intramedullary nailing may be used to correct deformity and prevent pain and refracture in FD of the bone of the lower limbs with large lesions, pathological fracture or deformities. All patients were allowed full athletic recovery following surgery.
\end{abstract}

\section{Introduction}

Fibrous dysplasia (FD) of the bone is a battery of tumorlike lesions in which fibrous tissue proliferation replaces normal bone architecture. FD accounts for only $5 \%$ of benign bone tumors (1). The incidence of this disease is 10-30 cases per million and accounts for $5.89 \%$ of benign

Correspondence to: Dr Xiaoqi Zhang, Department of Orthopedic Surgery, Anhui Provincial Hospital Affiliated to Anhui Medical University, 17 Lujiang Road, Hefei, Anhui 230001, P.R. China E-mail: xiaoqigk@sina.com

Key words: intramedullary nailing, fibrous dysplasia, lower limbs bone tumors in China. In 1891, FD was first described by Von Recklinghausen (2), but it was Lichtenstein (3) who labeled it polyostotic FD (PFD) in 1938. Lichtenstein and Jaffe (4) initially described the spectrum of the clinical, radiographic and histological symptoms. FD may involve a single skeletal site with an isolated lesion [monostotic FD (MFD)] or it may involve multiple bones [polyostotic FD (PFD)]. In addition, it may occur in association with distinct café-au-lait skin spots and/or a number of hyperfunctioning endocrinopathies labeled as McCune-Albright syndrome (MAS). In addition, FD may occur in association with intramuscular myxomas, which is referred to as Mazabraud's syndrome $(5,6)$. It has been reported that the monostotic form is 8-10 times more common than the polyostotic form (7). Compared with the spine, ribs and skull, FD is more common in the bones of the limbs. Following puberty or epiphyseal closure, the asymptomatic lesions of the majority of patients are restrictive and do not develop or progress. However, development and progression may occasionally be detected in lesions of patients with symptoms of pain, deformity and/or pathological microfracture.

In the present study, we retrospectively examined patient outcome following intramedullary nailing for FD of the lower limbs via long-term clinical follow-up.

\section{Patients and methods}

Patients. We retrospectively reviewed patients with FD of the lower limbs treated by intramedullary nailing between 2003 and 2010. A total of 39 patients participated in the study. The study was approved by the ethics committee of Anhui Medical University. Informed consent was obtained from the patients or the patient's family. The mean age of the patients was 31 years (range, 17-55), of which 22 were male and 17 were female. The mean follow-up period was 50 months (range, 4-93 months). Types of tumor included monostotic (33 patients) and polyostotic (7 patients). Symptoms included pain (15 patients), pathological fracture (10 patients), swelling/ deformity (13 patients), coxa vara (8 patients) and a limp (1 patient). Two patients had already undergone several treatments. One patient aged 12 years who presented with FD of the right humerus had received conservative treatment. Curettage and grafts of the left tibia were performed at 15 years old and were pathologically confirmed to be FD. Pathological fracture of the left tibia occurred at 17 years. Another patient presented with a lesion and received conservative treatment in 
Table I. Patient characteristics.

\begin{tabular}{|c|c|c|c|c|c|c|c|c|c|}
\hline Patient & $\begin{array}{c}\text { Age } \\
\text { (years) }\end{array}$ & Gender & Location & Symptoms & $\begin{array}{c}\text { Return to full } \\
\text { activity (months) }\end{array}$ & Guille $^{a}$ & Guille $^{\mathrm{b}}$ & $\begin{array}{c}\text { Neck shaft } \\
\text { angle }^{\mathrm{a}}\end{array}$ & $\begin{array}{c}\text { Neck shaft } \\
\text { angle }^{b}\end{array}$ \\
\hline 1 & 35 & $\mathrm{~F}$ & Femur (L) & Pain & 4.8 & & & & \\
\hline 2 & 44 & M & Bilateral limb & Fracture & 14.1 & & & & \\
\hline 3 & 42 & $\mathrm{~F}$ & Femur (R) & Pain & 5.1 & & & & \\
\hline 4 & 38 & M & Tibia (R) & Pain & 4.9 & & & & \\
\hline 5 & 31 & $\mathrm{~F}$ & Femur (R) & Pain & 4.2 & & & & \\
\hline 6 & 20 & M & Tibia (R) & Fracture & 3.8 & & & & \\
\hline 7 & 29 & M & Femur (L) & Fracture & 5.0 & & & & \\
\hline 8 & 26 & M & Femur (L) & Deformity & 5.8 & 3 & 9 & 70 & 119 \\
\hline 9 & 33 & M & Femur (R) & Pain & 3.9 & & & & \\
\hline 10 & 41 & M & Femur (R) & Deformity & 4.7 & & & & \\
\hline 11 & 35 & $\mathrm{~F}$ & Tibia (R) & Pain & 4.0 & & & & \\
\hline 12 & 17 & M & Tibia (R) & Fracture & 2.6 & & & & \\
\hline 13 & 18 & $\mathrm{~F}$ & Femur $(\mathrm{R})+$ skull & Deformity & 5.2 & 4 & 9 & 85 & 120 \\
\hline 14 & 45 & M & Tibia $(\mathrm{R})+\mathrm{L} 3$ & Pain & 3.6 & & & & \\
\hline 15 & 23 & M & Femur (L) & Deformity & 4.7 & & & & \\
\hline 16 & 30 & M & Femur (L) & Fracture & 5.4 & & & & \\
\hline 17 & 51 & $\mathrm{~F}$ & Tibia (L) & Pain & 6.4 & & & & \\
\hline 18 & 17 & M & Femur (L) & Pain & 4.4 & & & & \\
\hline 19 & 18 & $\mathrm{~F}$ & Femur (L) & Deformity & 3.5 & 5 & 10 & 104 & 130 \\
\hline 20 & 55 & $\mathrm{~F}$ & Femur $(\mathrm{R})+\mathrm{C} 6 \mathrm{C} 7$ & Pain & 6.8 & & & & \\
\hline 21 & 18 & M & Femur $(\mathrm{R})+$ tibia $(\mathrm{R})$ & Deformity & 3.2 & 3 & 9 & 75 & 120 \\
\hline 22 & 34 & M & Femur (L) & Deformity & 4.5 & 6 & 8 & 120 & 140 \\
\hline 23 & 20 & $\mathrm{~F}$ & Tibia (R) & Pain & 3.3 & & & & \\
\hline 24 & 18 & M & Femur (L) & Deformity & 5.5 & 5 & 8 & 90 & 127 \\
\hline 25 & 20 & M & Femur (R) & Fracture & 4.7 & & & & \\
\hline 26 & 30 & M & Femur (L) & Deformity & 5.8 & & & & \\
\hline 27 & 18 & $\mathrm{~F}$ & Femur (L) & Limp & 4.2 & & & & \\
\hline 28 & 48 & $\mathrm{~F}$ & Femur (L) & Fracture & 6.0 & & & & \\
\hline 29 & 54 & $\mathrm{~F}$ & Femur $(L+R)$ & Pain & 5.4 & & & & \\
\hline 30 & 20 & M & Femur (L) & Fracture & 3.6 & & & & \\
\hline 31 & 52 & $\mathrm{~F}$ & Femur (R) & Deformity & 6.5 & & & & \\
\hline 32 & 21 & $\mathrm{~F}$ & Femur $(\mathrm{L})+$ fibula $(\mathrm{L})$ & Pain & 3.2 & & & & \\
\hline 33 & 19 & $\mathrm{~F}$ & Femur (R) & Fracture & 2.4 & & & & \\
\hline 34 & 47 & M & Tibia (L) & Pain & 3.1 & & & & \\
\hline 35 & 21 & $\mathrm{~F}$ & Tibia (R) & Pain & 2.6 & & & & \\
\hline 36 & 33 & $\mathrm{~F}$ & Femur (L) & Deformity & 4.0 & 4 & 7 & 87 & 120 \\
\hline 37 & 43 & $\mathrm{~F}$ & Femur (R) & Deformity & 5.5 & 5 & 6 & 92 & 125 \\
\hline 38 & 23 & M & Tibia (L) & Deformity & 4.1 & & & & \\
\hline 39 & 52 & M & Tibia (L) & Fracture & 5.7 & & & & \\
\hline
\end{tabular}

aPreoperatively; ${ }^{\mathrm{b}}$ postoperatively; L, left; R, right; L3, lumbar 3; C6C7, cervical vertebra 6, cervical verterbra 7.

the neck of the left femur aged 10 years following a two-year limp. The patient underwent curettage due to deformity of the skull at 13 years old, and coxa vara was diagnosed at 18 years. The sites of the lesions were the femur (31 lesions), coxa vara (8 lesions), tibia (14 lesions), fibula (1 lesion), spine (2 lesions), bilateral lower limbs (1 patient), ipsilateral femur and tibia (1 patient) and ipsilateral femur and fibula (1 patient). The lesions located by patient self-observation and clinical manifestations, were detected by radiography, including plain films, computed tomography (CT) and magnetic resonance imaging (MRI). Following surgery, pathology was the ulti- mate diagnostic method. Patients characteristics are shown in Table I.

Surgical technique. The patient was placed in the supine position under an image intensifier. During surgery, a cortical fenestration sufficiently large to be able to visualize the whole lesion was made using an osteotome. The lesion was removed with curettes, and the cavity was enlarged with a burr and irrigated with sterile saline. The removed tissue was sent for pathological examination. For varus of the hips, a lateral closing wedge osteotomy was performed and a guide pin was 
A

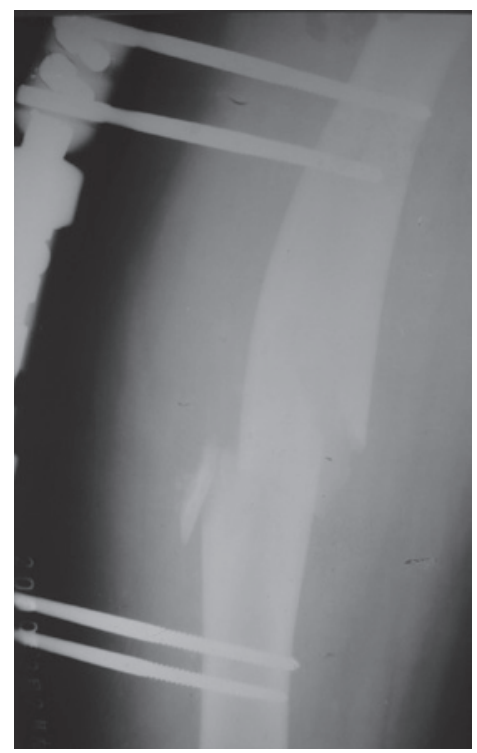

B

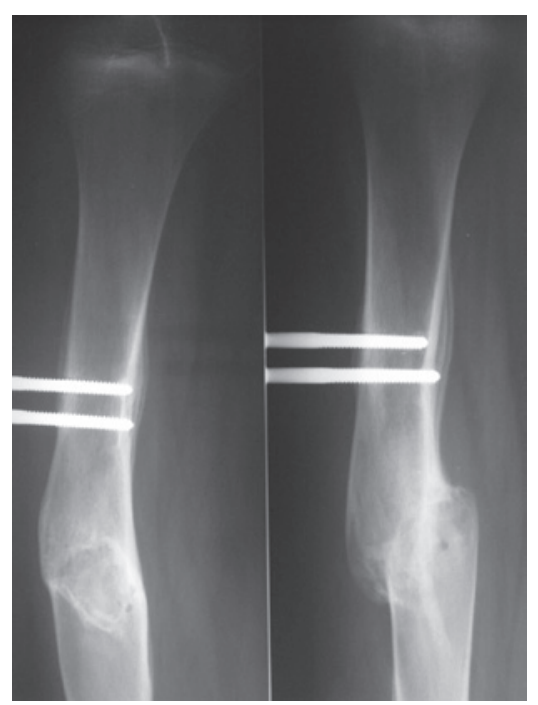

C

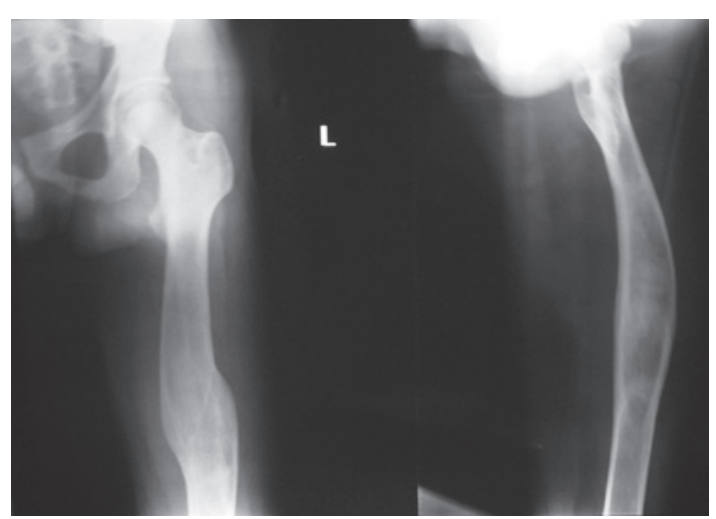

Figure 1. (A) Immediate postoperative, (B) 6-month and (C) 1-year follow-up radiographs of a 12-year-old male with a pathological fracture of the left femur that was treated with an external fixator.

inserted into the medullary canal through the distant osteotomized site. This was then reamed, and repeated removal of fibrous tissues of the intramedullary cavity was performed. After reaming, an intramedullary nail was inserted and then

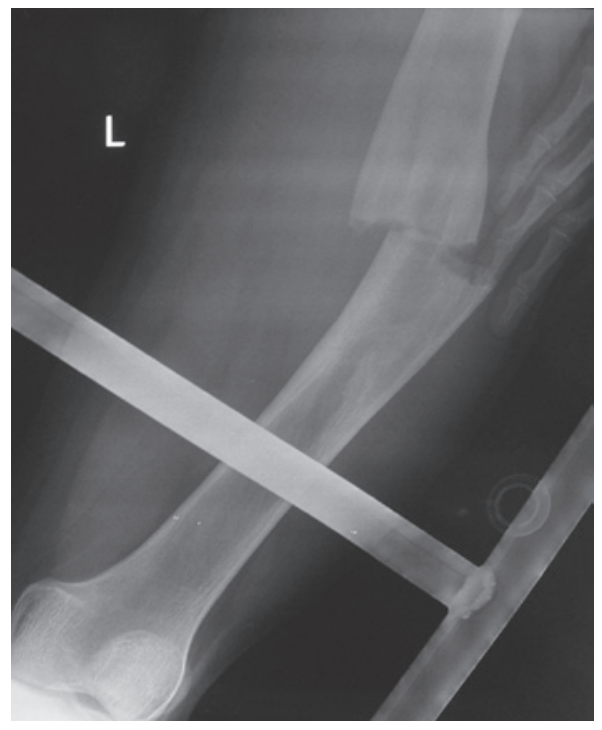

Figure 2. Refracture of the left femur after playing basketball at two years follow-up.

locked with proximal and distal screws. The cavity was then irrigated with sterile saline followed by the placement of a combination of autogenous cancellous bone and/or allograft cancellous chips.

Rehabilitation. Following surgery, all patients were advised against weight bearing but to mobilize joints depending on the location of the lesion. Patients were then provided with crutches to use for walking. Approximately two weeks after surgery, if there was clinically significant restrictive motion, physiotherapy was offered. Patients received a wound check and suture removal two weeks after surgery. Partial weight bearing was allowed approximately 2-3 months later. Time taken to return to normal walking was determined by patients' symptoms and follow-up radiographical imaging of osteotomy sites.

Follow-up. Patients were reexamined using plain film radiography following surgery. Radiography and clinical examinations were repeated every month for the first six months, and once every six months after that. Outcome criteria for the study included: i) pain status at the last follow-up; ii) time taken until return of full activity; iii) repeat/ additional surgical interventions. Pain was measured using a patient visual analogue scale. Recovery time was measured between the date of surgery and the date when full activity was resumed. The clinical score was in accordance with the modified criteria of Guille et al (8).

\section{Results}

Recovery time. None of the patients had infection, thromboembolism or other notable complications. No loosening of screws or refracture was detected in the follow-up period. However, one patient with a lesion in the bilateral lower limbs, who underwent an osteotomy of the femur, had an unrelated pathological fracture on the other femur and was treated with 
A

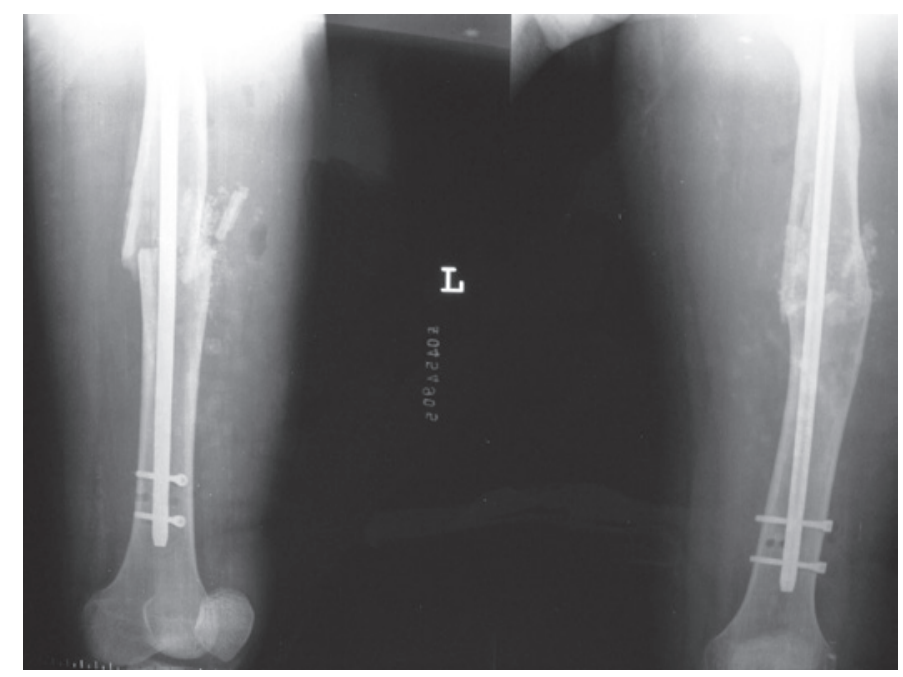

B

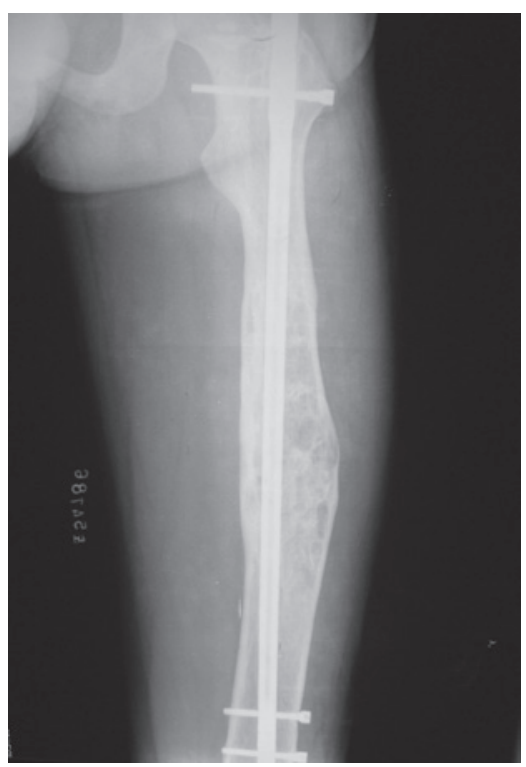

C

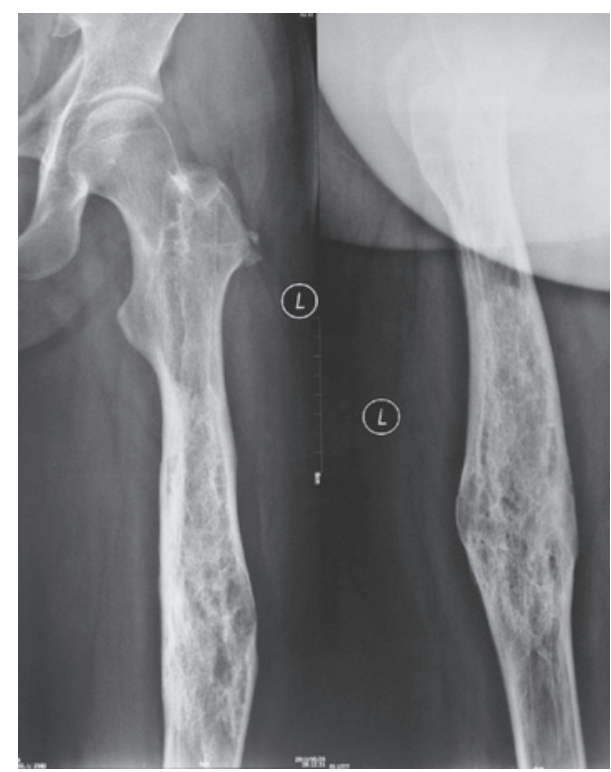

Figure 3. (A) Immediate postoperative, (B) 1-year and (C) 3-year follow-up radiographs of intramedullary nailing, curettage and bone grafting of the left femur.

further intramedullary nailing surgery. Therefore, the time taken to return to full activity was prolonged. One patient aged 12 years presented with a pathological fracture of FD and was treated with an external fixator (Fig. 1A). The fracture resulted in a malunion as demonstrated in the six-month and one-year follow-up radiographs (Fig. 1B and C, respectively). A refracture of the lesion (Fig. 2) was detected and treated with intramedullary nailing at two years follow-up (Fig. 3A). No loosening of screws or refracture was detected in the follow-up period (Fig. 3B). The fixator was removed at three years follow-up (Fig. 3C).

Patient recovery and symptoms. A total of 32 patients (82.1\%) presented with no pain and 7 patients $(17.9 \%)$ had mild and occasional pain at the last follow-up. All patients resumed full activity and function. One patient took longer to return to full activity due to pathological fracture of another limb. The clinical score was in accordance with the modified criteria described by Guille et al (8). The clinical score improved from an average of 4.4 (range, 3-6) points prior to surgery to an average of 8 (range,
6-10) points at the last follow-up. The neck shaft angle of the femur was corrected from an average of $90^{\circ}$ (range, $70-120^{\circ}$ ) prior to surgery to an average of $125^{\circ}$ (range, $119-140^{\circ}$ ).

\section{Discussion}

FD of the bone is a type of benign bone lesion. A single skeletal site with an isolated lesion is involved in the majority of patients, but the disease may be polyostotic. Previous studies have not reported indicators for FD, although there are several methods of treatment, including conservative treatment (medications and braces) and surgical procedures (curettage, curettage and graft, and internal fixation). Kusano et al (9) revealed that the majority of lesions of FD usually cease to progress or develop following adolescence, with the exception of McCune-Albright syndrome. Therefore, conservative treatment is recommended for adolescents. The mass of the lesion may not require surgery, unless ongoing pain, lesion expansion or bone percentage predisposes the bone to pathological fracture or a fracture has already occurred (10-15). Pain is associated with lesion 
expansion. Generally, curettage of the lesion leaves a cavity which predisposes the bone to destabilization or even pathological fracture. Therefore, an external or internal fixation is performed for stabilization. In PFD patients, particularly those with shepherd's crook deformity, curettage and bone grafting are not advised due to reabsorption of bone graft and lesion expansion following surgery. In this case, internal fixation and correction are recommended for patients with distinct deformity and/or pathological fracture, which result in poor quality of life and complications due to bed rest.

In the case of large lesions (more than two-thirds of the bone) or upon enlargement of the lesion, the bone becomes unstable and predisposed to fracture when a cavity is left following curettage. Muscle atrophy, thromboembolism, ankylosis or other notable complications may occur in the long-term external fixation period. However, it is difficult to provide sufficient stability with plates and screws in the vicinity of the weakened bones, and fracture or refracture may occur due to the stress-shielding effect of the distal part of the plate. Guille et al (8) suggested that a refracture may easily occur after the plate and screws are removed in patients with a lesion involving a large area. O'Sullivan and Zacharin (16) reported that intramedullary nailing and bisphosphonate treatment of 10 femurs with McCune-Albright syndrome prevented fractures and resulted in improved walking. Thus, we consider that intramedullary nailing may be used in other anatomical sites, with the exception of proximal femoral lesions of the lower limbs. This approach results in fewer sequelae at the site of the lesion or fracture than when using plates and screws, and provides sufficient stability with fixation in the normal distal bones. Particularly in cases with a lesion covering a large area, the incision has to be enlarged to fit a long metal plate due to the vicinity of weakened bones, and loss of fixation, delayed union or non-union of the fracture may occur more frequently. When a valgus osteotomy is performed, overcorrection of the neck shaft angle is recommended (more than $130^{\circ}$ ) in anticipation of postoperative loss of valgus in lesions of the proximal femur, including shepherd's crook deformity. However, a satisfactory clinical result can be expected when the neck shaft angle is at least $90^{\circ}$ (8). We recommended overcorrection of the neck shaft angle in anticipation of loss of valgus angle after surgery, which is limited by muscle contracture between the greater trochanteric area and the ilium. This approach is prevented in adolescents due to epiphyseal injuries. However, intramedullary nailing is performed on the occurrence of epiphysis fusion or marked changes of the epiphysis.

During surgery, the cortical fenestration should be sufficiently large to allow visualization of the whole lesion with an osteotome. Due to the internal fixation, the bone was stable and the cortical fenestration did not result in pathological fracture during or following surgery. We recommend removal of the lesion using curettes and enlargement of the cavity using a burr. However, normal trabecular bone reduction due to repeated curettes may cause delayed union or non-union of the fracture.

A number of authors have recommended that the defect should be filled with bone grafts or substitutes following curettage. Autogenous bone grafting is recommended due to the advantages of lack of immunological reaction and successful bone induction. However, obtaining sufficient bone for large cavities may be difficult and sequelae may be generated at the donor site. In addition, certain authors have recommended curettage of benign bone tumors without grafts (17). We recommend curettage and grafting with autogenous bone from the ilium. However, large defects could alternatively be filled with substitutes including cement, hydroxyapatite or tricalcium phosphate, as bone grafts are difficult to obtain.

In conclusion, indicators for FD of the bone have not yet been established due to low incidence and small sample sizes. However, we propose an approach which allows patients to return to full, painless activity quickly in the majority of cases. The approach used in this study is recommended as a reliable and effective surgery for FD of the lower limbs.

\section{References}

1. Campanacci M (ed): Bone and Soft Tissue Tumors: Clinical Features, Imaging, Pathology, and Treatment. 2nd edition. Springer, New York, NY, 1999.

2. Von Recklinghausen F: Die Fibrose oder deformierende Ostitis, die Osteomalacie und die oesteoplastische carcinose in ihren gegenseitigen Beziehungen. Festschrift Rudolf Virchow zum 13. Oktober, Berlin, 1891.

3. Lichtenstein L: Polyostotic fibrous dysplasia. Arch Surg 36: 874-898, 1938.

4. Lichtenstein L and Jaffe HL: Fibrous dysplasia of bone: a condition affecting one, several or many bones, graver cases of which may persent abnormal pigmentation of skin, premature sexual development, hyperthyroidism or still other extraskeletal abnormalities. Arch Pathol 33: 777-816, 1942.

5. Wirth WA, Leavitt D and Enzinger FM: Multiple intramuscular myxomas. Another extraskeletal manifestation of fibrous dysplasia. Cancer 27: 1167-1173, 1971.

6. Blasier RD, Ryan JR and Schaldenbrand MF: Multiple myxomata of soft tissue associated with polyostotic fibrous dysplasia. A case report. Clin Orthop Relat Res 206: 211-214, 1986.

7. Dorfman HD and Czerniak B (eds): Bone Tumors. Mosby, St. Louis, MO, 1998

8. Guille JT, Kumar SJ and MacEwen GD: Fibrous dysplasia of the proximal part of the femur. Long-term results of curettage and bone-grafting and mechanical realignment. J Bone Joint Surg Am 80: 648-658, 1998.

9. Kusano T, Hirabayashi S, Eguchi T and Sugawara Y: Treatment strategies for fibrous dysplasia. J Craniofac Surg 20: 768-770, 2009.

10. DiCaprio MR and Enneking WF: Fibrous dysplasia. Pathophysiology, evaluation, and treatment. J Bone Joint Surg Am 87: 1848-1864, 2005.

11. Easley ME and Kneisl JS: Pathological fractures through large nonossifying fibromas: is prophylactic treatment warranted? J Pediatr Orthop 17: 808-813, 1997.

12. Arata MA, Peterson HA and Dahlin DC: Pathological fractures through non-ossifying fibromas. Review of the Mayo Clinic experience. J Bone Joint Surg Am 63: 980-988, 1981.

13. Drennan DB, Maylahn DJ and Fahey JJ: Fractures through large non-ossifying fibromas. Clin Orthop Relat Res 103: 82-88, 1974.

14. Ahn JI and Park JS: Pathological fractures secondary to unicameral bone cysts. Int Orthop 18: 20-22, 1994.

15. Betsy M, Kupersmith LM and Springfield DS: Metaphyseal fibrous defects. J Am Acad Orthop Surg 12: 89-95, 2004.

16. O'Sullivan M and Zacharin M: Intramedullary rodding and bisphosphonate treatment of polyostotic fibrous dysplasia associated with the McCune-Albright syndrome. J Pediatr Orthop 22: 255-260, 2002.

17. Yanagawa T, Watanabe $H$, Shinozaki $T$ and Takagishi $K$ : Curettage of benign bone tumors without grafts gives sufficient bone strength. Acta Orthop 80: 9-13, 2009. 